\title{
High performance optical free-space links for quantum communications
}

Matthias Goy, René Berlich, Andrej Kržič, Daniel Rieländer, Teresa Kopf, et al.

Matthias Goy, René Berlich, Andrej Kržič, Daniel Rieländer, Teresa Kopf, Sakshi Sharma, Fabian O. Steinlechner, "High performance optical free-space links for quantum communications," Proc. SPIE 11852, International Conference on Space Optics - ICSO 2020, 118520 (11 June 2021); doi: $10.1117 / 12.2599163$

SPIE Event: International Conference on Space Optics - ICSO 2021, 2021, Online Only 


\section{International Conference on Space Optics-ICSO 2020}

Virtual Conference

30 March-2 April 2021

Edited by Bruno Cugny, Zoran Sodnik, and Nikos Karafolas
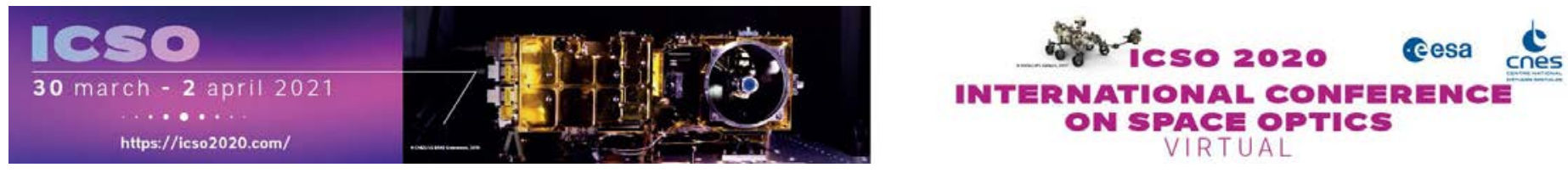

\section{High performance optical free-space links for quantum communications}

\section{Cesa isopueations ecnes}

International Conference on Space Optics - ICSO 2020, edited by Bruno Cugny, Zoran Sodnik, Nikos Karafolas, Proc. of SPIE Vol. 11852, 118520I - (c) 2021 ESA and CNES

CCC code: $0277-786 \mathrm{X} / 21 / \$ 21 \cdot$ doi: $10.1117 / 12.2599163$ 


\title{
High Performance Optical Free-Space Links for Quantum Communications
}

\author{
Matthias Goy ${ }^{\mathrm{a}}$, René Berlich ${ }^{\mathrm{a}}$, Andrej Kržič̃a ${ }^{\mathrm{a}}$, Daniel Rieländer ${ }^{\mathrm{a}}$, Teresa Kopf ${ }^{\mathrm{a}}$, Sakshi \\ Sharma ${ }^{\mathrm{a}}$, and Fabian Steinlechner ${ }^{\mathrm{a}, \mathrm{b}}$ \\ ${ }^{a}$ Fraunhofer Institute for Applied Optics and Precision Engineering, Jena, Germany \\ ${ }^{\mathrm{b}}$ Abbe Center of Photonics, Friedrich Schiller University Jena, Germany
}

\begin{abstract}
Secure communication networks are the critical infrastructure of the information age. To ensure secure communication between governmental institutions and other high-security environments, the German Federal Ministry of Education and Research (BMBF) initiated an ambitious project - the QuNET initiative. In a joint effort, the Max Planck Institute for the Science of Light (MPL), the German Aerospace Center (DLR) and the Fraunhofer Society aim to develop the technological basis of a German quantum key distribution (QKD) infrastructure. This paper describes the infrastructure used in a first link demonstrator within this project and how we achieved the transition from initial quantum transceiver concepts to first link experiments.
\end{abstract}

Keywords: quantum communications, experimental platforms, QuNET, QuBus, obscuration-free telescope

\section{INTRODUCTION}

The QuNET initiative aims to establish the physical-technical basis of quantum communication and link technologies for application in a German high-security network. Amongst other topics, research and development will address quantum key distribution (QKD) in hybrid networks in multi-user operation with simultaneous transmission of classical user data as well as the development of quantum communications hardware for fiberbased and free-space quantum communication links that can be implemented in and alongside classical communication infrastructure. In the first phase of the QuNET initiative the partners will perform several QKD field demonstrations in fiber and free-space and gauge the benefits of a multitude of quantum state encoding schemes, protocols, and operational wavelengths. Here, we report on the progress towards a demonstration of polarization-entanglement-based free-space quantum communications in the Si-wavelength band. Specifically, we describe the development of required optical hardware as enabling tools for ambitious future quantum communication projects. These are integrated in two transportable terminal types. First, the PhotonBox - an optical transceiver designed for a terrestrial free-space link testbed in the city of Jena and second, the QuBus - a mobile open science platform for a variety of quantum communication field trials. For both cases, Fraunhofer IOF has developed a mobile telescope-board, which is tailored to the specific requirements of free-space quantum communication links through turbulent atmosphere. The PhotonBox transceiver is equipped with a large-aperture $(200 \mathrm{~mm})$ obscuration-free folded mirror telescope for optimal link transmission in the two most common wavelength bands for free-space quantum links: $780-850 \mathrm{~nm}$ and around $1550 \mathrm{~nm}$. Both transmitter- and receiver terminals are equipped with fast beam steering systems for the correction of angle-of-arrival fluctuations and beam-wander arising from atmospheric turbulence. The telescope-boards of the PhotonBox -link are attached to motorized mounts which can move in azimuth and elevation. With the help of guiding LEDs, cameras and an image analysis algorithm, a fully automated alignment of both telescope systems is ensured. The PhotonBox will enable a variety of experimental field trials in Jena, in both the quantum and classical domain. Meanwhile, the QuBus development aims to provide an open science platform for all German and European institutions that work on quantum communication technologies. In pursuit of the vision of providing a versatile quantum science platform, the QuBus combines state-of-the-art laboratory infrastructure with advanced optical link technology in a highly mobile, robust and thermally controlled shipping container that can be installed with minimum effort

Further author information: (Send correspondence to M. Goy)

M.Goy: E-mail: matthias.goy@iof.fraunhofer.de, Telephone: +49 3641807120 
at field trial locations. The heart of this platform is the telescope-board with fast tip-tilt correction. It uses standardized optical and electrical interfaces and mounting racks for modular integration of quantum communication hardware. Flexible coarse alignment of the transmitter and receiver systems (QuBus-Alice and QuBus-Bob) is ensured by a motorized periscope system. The QuBus open science platform will thus allow for a wide range of future field trials, in particular experiments with optical links to and from air planes, high altitude platforms, and - ultimately - satellites.

\section{LINK INFRASTRUCTURE FOR QUANTUM COMMUNICATION FIELD TESTS}

The development of multi-purpose experimental terminals for quantum communications was one ambitious goal of the QuNET project. Experiments that should be performed in the first phase of QuNET as well as those of the second phase and also from other projects can be realized with telescope breadboards that enable an easy integration and operating of transmitter and receiver modules. The telescope breadboard, which is described in section 3, is designed for the use with our two main experimental terminals: PhotonBox and QuBus. Beside source and detection devices for quantum experiments both terminals are equipped with classical communication systems.

\subsection{PhotonBox - a low-cost lab container}

The PhotonBox is a low-cost container laboratory and part of $1.7 \mathrm{~km}$ intra-city link in Jena, Germany. It is built from a standard 10-foot shipping container that was lifted onto the roof (6th floor) of the local energy provider. Figure 1 shows the floor plan of the PhotonBox as well as a photograph of the outside view. The container has one door, one front window which is equipped with a $450 \mathrm{~mm}$ x $450 \mathrm{~mm}$ optical fused silica substrate and one window with an electrical shutter on the roof. Electricity, which must be provided from an external source as well as internet connection is feed into the container through a sealed hole. For an easy access 50 sockets are distributed around the walls. An air-condition together with 100-mm-thick insulation ensure room temperature with acceptable stability also at high or low outside temperatures. Basis for the experiments is a science desk
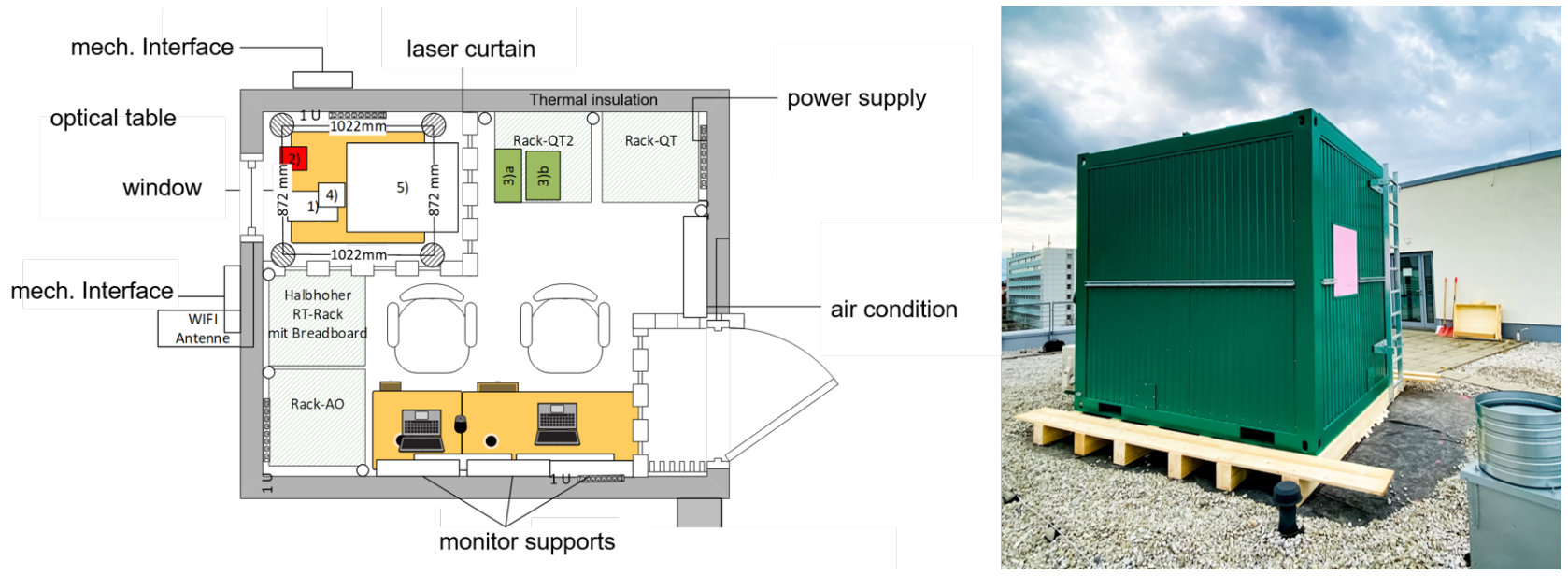

Figure 1. Drawing of the PhotonBox floor plan and a photograph from outside, while installation on the roof of the building.

or an optical table close to the front window. The desk with a size of $1000 \mathrm{~mm} \times 900 \mathrm{~mm}$ is decoupled from the containers floor to prevent vibration or any swinging effect when operators move while an experiment is running. The legs from the desk are extended with aluminium posts that are feet through four wholes in the containers floor. Thus, the legs can be positioned directly on the roof the building. The remaining space inside is filled with racks for the quantum communications equipment and two computer workstations. 


\subsection{QuBus - The concept of a high performance mobile platform for quantum communications}

The QuBus is a more sophisticated version of the PhotonBox which is described in section 2.1. While the PhotonBox is developed as a low-cost laboratory providing the basic laboratory infrastructure like appropriate humidity, temperature, electricity and internet, the QuBus is designed as a robust experiment vehicle for ad-hoc field measurement campaigns in quantum communications. Basis for the QuBus is a 15-foot shipping container that can be transported by a low-bed trailer. Main difference to the PhotonBox is how the light of the experiments is feed out of the container and directed to the target. In the PhotonBox, the light coming from the telescope breadboard must be directed by moving the breadboard itself. This is done by using a commercially available motorized mount (see section 3.1). In contrast, in the QuBus the telescope breadboard and all the optical and electrical equipment can be fixed onto an optical table rigidly, which reduces limitations in weight and geometry of the equipment and enables a faster change of all devices even while operation. The light is then directed by a highly stable, precise periscope mechanics on the roof of the QuBus (see Figure 2). While the coarse alignment of the beam coming from the PhotonBox must be done by positioning the container itself in the right way, the alignment of the QuBus' beam is totally independent from the vehicles orientation. The periscope enables a $-5^{\circ}$ to $90^{\circ}$ angle in altitude and a $360^{\circ}$ angle in azimuth. The diameter of the periscope is $250 \mathrm{~mm}$ and matches the aperture of the telescope of the breadboard described in section 3.

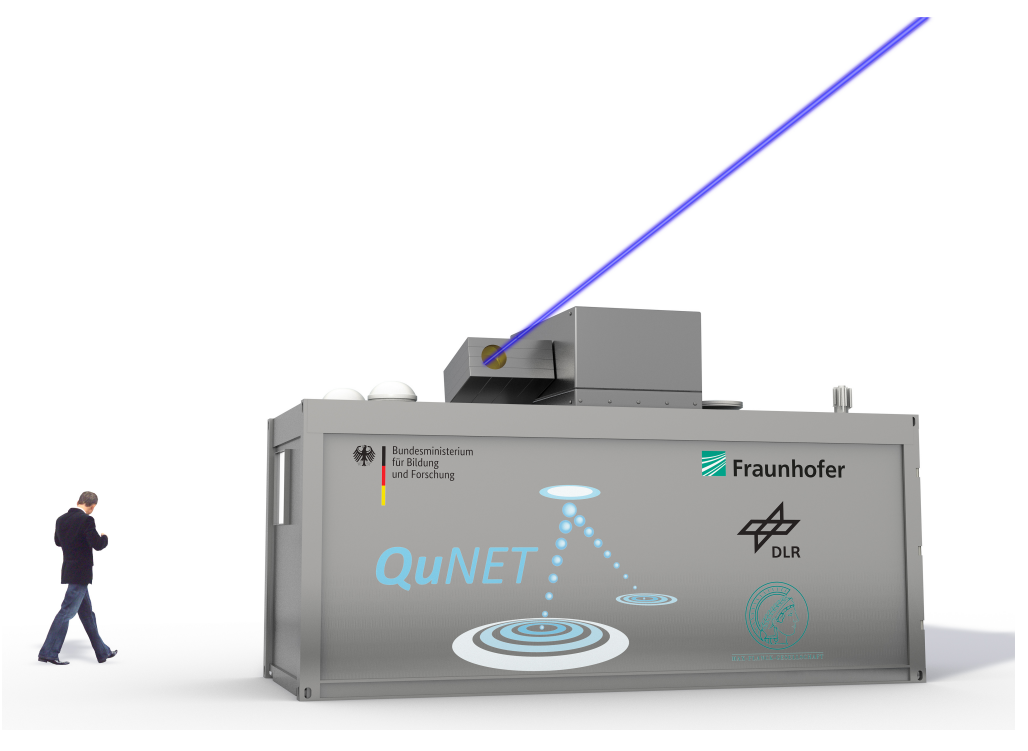

Figure 2. 3D-Model of the QuBus. View from outside.

For secure transportation operation, the entire equipment inside and outside the container is installed according to military standards. The QuBus is currently under construction. First field tests are planned for summer 2022 .

\section{MULTI-WAVELENGTH TRANSCEIVER PLATFORM}

The multi-wavelength transceiver system was designed and implemented as a multi-purpose platform for experiments in quantum communications. It contains an opto-mechanical transmitter (ALICE) and receiver (BOB) module, multiple quantum sources at $810 \mathrm{~nm}$ and $1550 \mathrm{~nm}$, as well as multiple QKD analysis modules as depicted in the schematic overview in Figure 3.

The individual subsystems are detailed in the following subsections and the optical and mechanical design, the beam stabilization means, as well as the concept of the sources and analysis modules that have been used for the first experiments are described. 


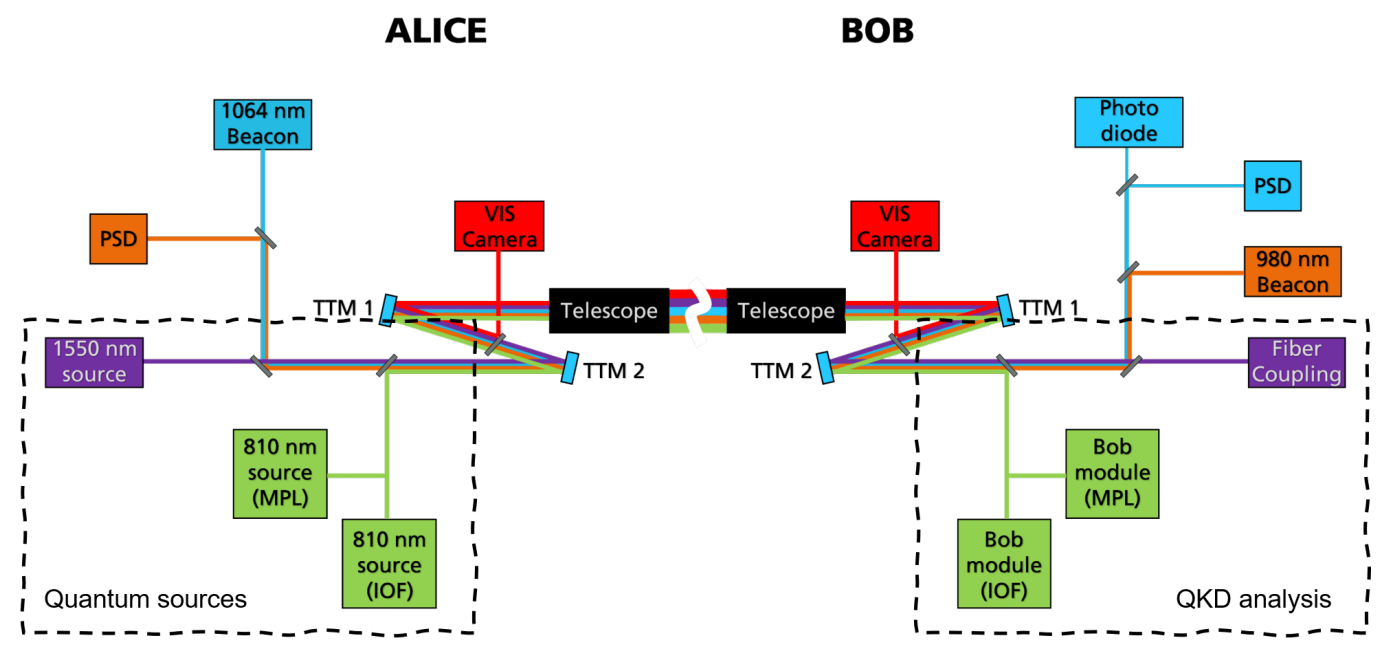

Figure 3. Schematic diagram of the multi-purpose transceiver platform components.

\subsection{Opto-mechanical transmitter and receiver module}

The opto-mechanical transmitter and receiver modules consist of two distinct modules. The optical beam paths inside the two modules are shown in Figure 3. The basis of each module is an off-the-shelf $750 \mathrm{~mm} \times 750 \mathrm{~mm}$ optical breadboard braced by aluminium profiles. The modules provide the basic infrastructure for flexible quantum experiments and therefore include numerous functionalities.

Each module includes an obscuration-free, off-axis mirror telescope, which provides a large transmitting and receiving aperture with a diameter of $200 \mathrm{~mm}$, respectively. Each telescope is characterized by a 20x optical magnification and features multiple aspherical mirror surfaces with a protected gold coating. The respective design is optimized to provide a diffraction limited imaging performance over a large field of view up to $3.5 \mathrm{mrad}$. In addition, the individual mirror surfaces provide a minimized angle of incidence variation across their aperture in order to minimize their effect on the polarization state of the laser beams. The ultra-precision manufactured mirrors are integrated in a housing, manufactured from aluminum alloy and containing pattern of ribs for bracing purposes. The housing is manufactured from a single piece, providing highly precise reference surfaces for the mirrors. Three bipods mount the housing strainlessly to the breadboard. In addition to their main telescope, each module includes an active $4 \mathrm{D}$ beam stabilization system. This adaptive optical system corrects for the influence of atmospheric turbulences during the laser beam propagation between the transmitting and receiving aperture, i.e. tip/tilt wavefront aberrations as well as optical beam wander. The system consists of two tip-tilt mirrors (TTMs), which are based on commercially available, ultra-fast piezoelectric platforms from Physik Instrumente. The plattforms are integrated into a customized mirror mount that provides the needed dynamic stiffness. The TTMs are controlled in a closed loop using a commercially available control system (TEM Aligna). The system utilizes the signal of a set of two position sensitive devices (PSDs). The PSDs measure the position and angle of an incoming beacon laser beam in order to stabilize the communication beam. The control loop provides a low response time of less than two milliseconds to counteract the rapidly changing wavefront aberrations. The telescope's bipods as well as the TTM mounts are screwed directly into the breadboard since this provides the needed stability.

As can be seen in Figrue 4, the telescope and the beam stabilization in the transmitter and the receiver module are followed by a combination of broad-band, high-pass, low-pass and narrow-band beam splitters. This combination facilitates the separation of the different beam paths depending on their wavelength in order to feed the different interfaces for the quantum sources and analysis equipment. A live-view camera for coarse subsystem alignment at $653 \mathrm{~nm}$ is implemented using a long-pass beam splitter located between the two TTMs. After the second TTM, the beam paths for the quantum source and the quantum receiver module at $810 \mathrm{~nm}$ and $1550 \mathrm{~nm}$, respectively, the PSD as well as a photo diode for the time synchronization at $970 \mathrm{~nm}$ or $1064 \mathrm{~nm}$, respectively, are isolated according to the schematic overview in Figure 3. Note that the transmission properties 


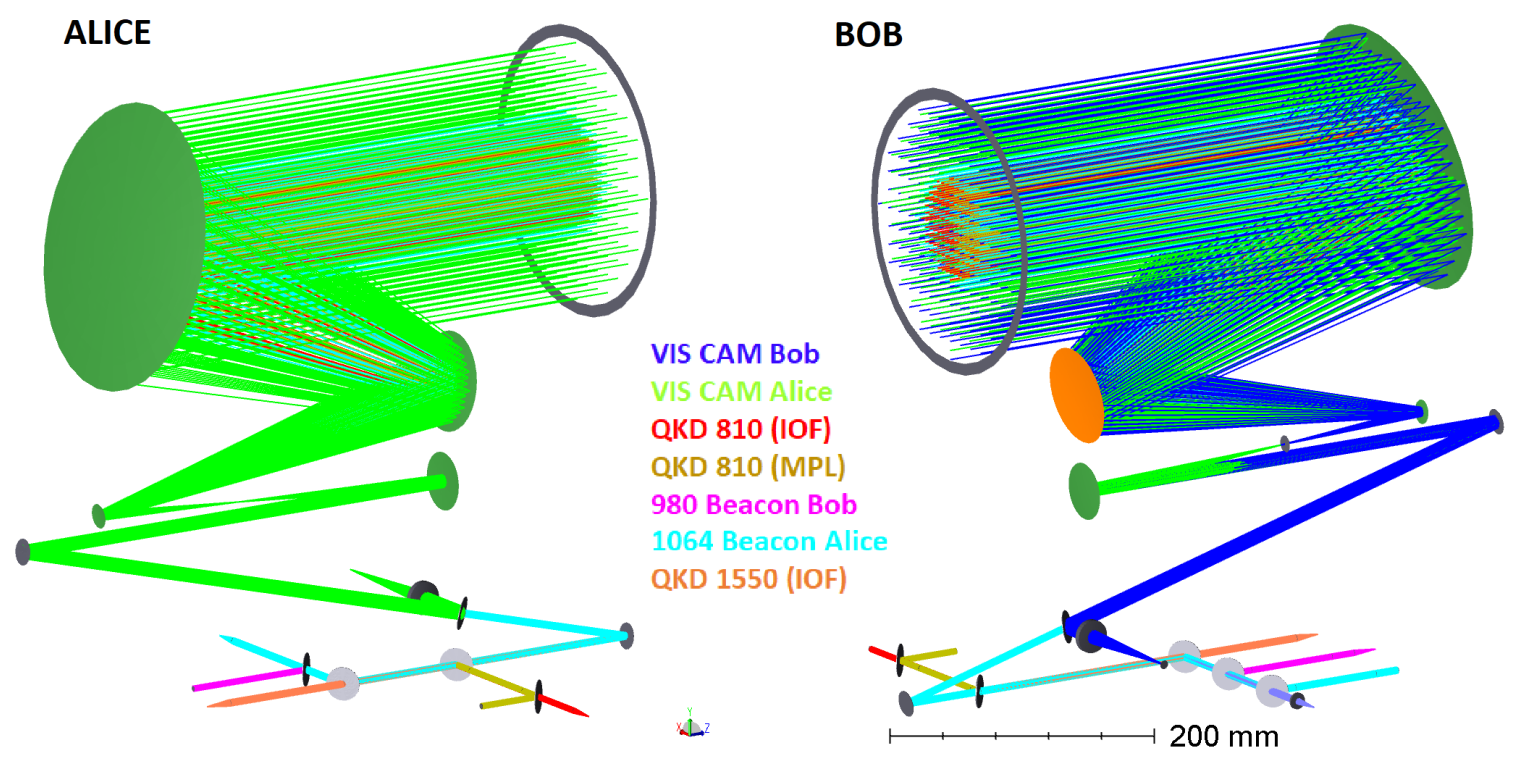

Figure 4. 3D beam path arrangement of a transmitter (ALICE) and receiver (BOB) module.

of all beam-splitting elements are optimized for a minimum polarization sensitivity at the QKD wavelengths. The respective opto-mechanical components can be flexibly arranged depending on the particular experiment using the standard bore pattern of the optical breadboard. The combination of a multitude of functionalities with respect to the QKD wavelengths requires a large installation space, which we reduced by using a 3D beam path arrangement. After passing the mirror telescope and the first TTM, the light is guided through an elongated hole in the telescopes housing and the breadboard. In doing so, both sides of the breadboard can be fully used and the overall assembly is kept compact. To prevent the breadboard from bending under the heavy weight, a frame of aluminum profiles and one additional profile on the opposite side of the ASA mount interface braces the breadboard. Four legs can be added to both sides of the frame, respectively, to align the optical system on an optical table. For a reliable functionality of the breadboard mount, the center of gravity (COG) of the moved mass must be close to its spin axis. Since the position of the COG depends on the chosen setup, we integrated an adjustable mechanism to balance the breadboard over the breadboard mount. One of the two transceiver systems with the the mounted telescope breadboard is shown in Figure 5.

\subsection{Quantum Sources}

Entangled photon pairs are generated via spontaneous parametric down-conversion (SPDC) in a nonlinear crystal. Here one can imagine that pump photons from a continuous-wave laser spontaneously decay into a pair of photons with lower energy. Energy- and momentum conservation lead phase matching conditions. These are acquired by a specific crystal-design with periodically poling and temperature adjustment. The temperature stabilized non-linear crystal is placed into a Sagnac-loop, in this configuration polarization entangled photon pairs can be created very efficiently. The specific source used for the free-space link creates pairs with a rate of $70 \mathrm{kHZ}$ at a wavelength of $810 \mathrm{~nm}$. The quality of the entanglement can be estimated by the visibilities the different polarization bases $\mathrm{H} / \mathrm{V}$ and $\mathrm{D} / \mathrm{A}$ with the present source values of $98.2 \%$ and $98.6 \%$ can be reached respectively. Further details about the source can be found in. ${ }^{1}$

\subsection{Receiver Modules}

The receiver for wavelengths around $810 \mathrm{~nm}$ is designed for polarization encoded quantum communication, compatible with most standard QKD protocols such as BB84 ${ }^{2}$ and BBM92. ${ }^{3}$ These protocols require polarization measurements in two mutually unbiased bases, namely horizontal-vertical (HV-basis) and diagonal-antidiagonal (DA-basis), and the selection of the basis for each incoming photon needs to be randomly selected. In our 


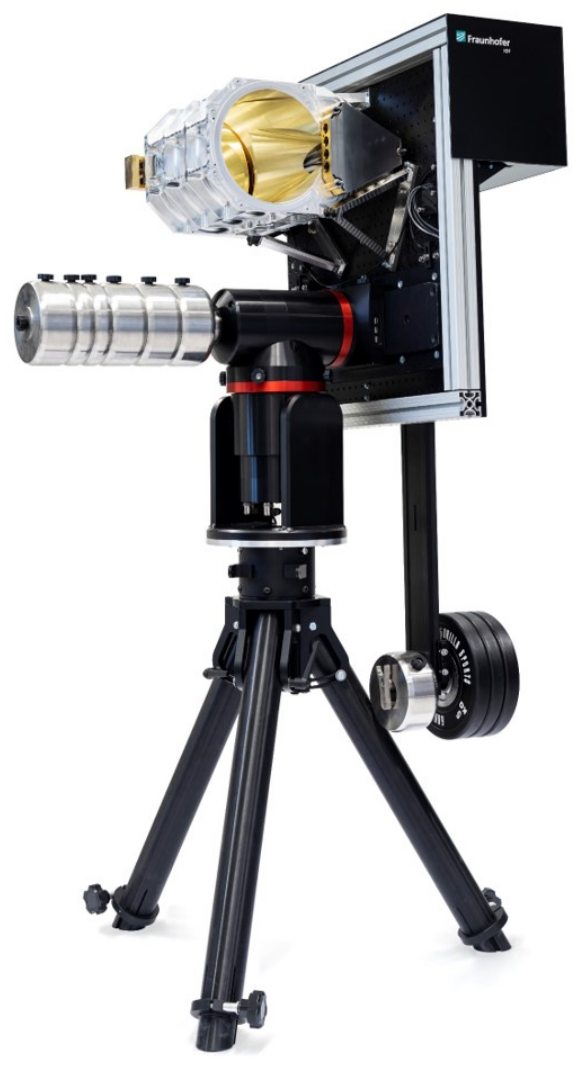

Figure 5. Image of the fully integrated Telescope Breadboard.

polarization analysis module (see Fig. 6), this is passively realized with a beam-splitter, of which one output is directly followed by a Wollaston prism to split $\mathrm{H}$ - and V-polarized photons, while the half-wave plate at the other output first rotates polarization by $45^{\circ}$, making the subsequent Wollaston prism split D- and A-polarized photons. Each of the four detection paths is finally coupled into a multi-mode fibre, which guides the light from the telescope board to the four single-photon avalanche detectors (SPADs) located in the rack.

To ensure matching of polarization coordinate systems at transmitter and receiver, a motorized polarization controller consisting of a stack of half- and quarter-wave plates was developed and integrated into the module, allowing for automatic basis alignment. Furthermore, the module incorporates two exchangeable filter slots that can be used to seamlessly replace the desired filter combination to match the wavelength of the source. Strong rejection of other wavelengths is of utmost importance, since the signal levels may be as low as in the order of 105 photons per second, amounting only to tens of femtowatts of power at $810 \mathrm{~nm}$. For the wavelengths around $1550 \mathrm{~nm}$, a rather different approach was chosen. These wavelengths are particularly interesting for the fibre-based quantum communication systems. For this reason, instead of directly performing polarization mode projection in free-space, the light is coupled into a single-mode fibre, opening the possibility to use the QuNET system as an interface between a free-space and a fibre-based communication channel. Once the light is coupled, it can be guided to any analysis system, allowing also for other encoding-schemes and protocols. 


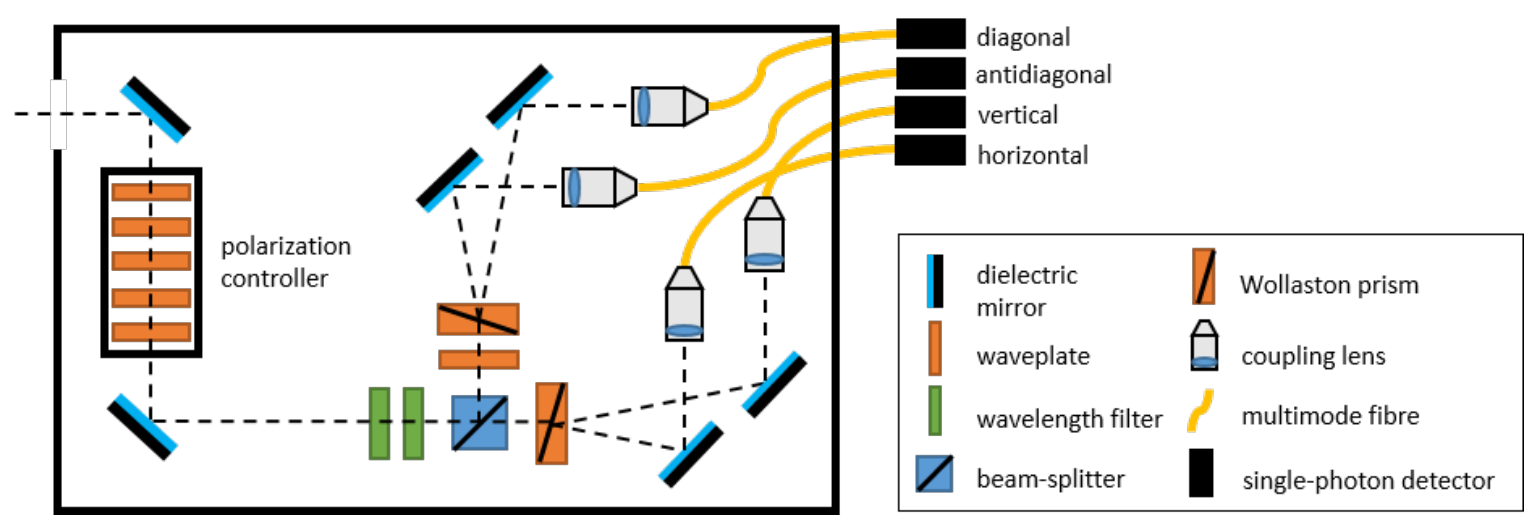

Figure 6. Polarization analysis module. Incoming photons are randomly measured either in horizontal-vertical or in diagonal-antidiagonal basis. Polarization controller ensures matching of transmitter and receiver polarization coordinate systems.

\subsection{Active beam stabilization}

Active beam stabilization is realized by utilizing a combination of two silver mirrors along the beam path that are mounted on fast piezo-based tip/tilt platforms. Together with a detection module at the end of the beam path, which provides the feedback signal for the piezo drivers, they form a closed-loop system. The combined control of the two mirrors provides a 4-dimensional beam stabilization in angle and position. The detection module consists of a beam splitter and two position-sensitive-detectors - one directly measures the centroid of the beam, while the other one positioned behind a lens in turn measures the angle of the beam. The system was designed to cope with beam wander and angle-of-arrival fluctuations induced by turbulences with Fried parameter of about $\mathrm{r}_{0}=2 \mathrm{~cm}$. The bandwidth of the entire correction system is estimated to be about $250 \mathrm{~Hz}$. These specifications are expected to suffice for beam stabilization in most conditions of the Jena free-space link. The system was tested on an actual turbulent $50 \mathrm{~m}$-link by running a measurement from $6 \mathrm{pm}$ until 10 am the next day. In addition to varying turbulence strength, the system was also exposed to relatively large but slow temperature changes, characteristic of a late November night in Jena. Figure 7 shows the four piezo drive signals used to control tilts of the mirrors (top row), and the corresponding residual beam angle/position error (bottom row). The solid black lines represent 5 -minute moving averages, indicating long-term changes in the system beam path. It can clearly be seen from piezo driver signals that the system mostly stayed well within its dynamical range of \pm 10 (abstract units) over the course of $16 \mathrm{~h}$, compensating for short-term turbulence effects as well as for long-term thermal drifts. Standard deviations (STDs) of the residual errors throughout the test are shown in Table 1. Note that these values correspond to angles and positions on the telescope breadboard - in order to translate them to the free-space link in between the two telescopes, angles need to be demagnified and positions magnified by 20 , which is the magnification factor of the telescopes .

Table 1. Standard deviations of residual beam angle and position errors on the telescope breadboard during the $16 \mathrm{~h}$ overnight measurement.

\begin{tabular}{|c|c|c|c|}
\hline angle $\mathrm{x} / \mu \mathrm{rad}$ & angle $\mathrm{y} / \mu \mathrm{rad}$ & position $\mathrm{x} / \mu \mathrm{m}$ & position $\mathrm{y} / \mu \mathrm{m}$ \\
\hline 9.2 & 6.8 & 26.7 & 9.7 \\
\hline
\end{tabular}

\section{FIRST RESULTS OF A SHORT LINK TEST}

The functionality of the QKD-system has been shown on short free space link in between two buildings buildings of Fraunhofer IOF. Polarization-entangled photons (photon A and photon B) are generated in the source described in the preceding section. Photon A is sent to Alice via an optical single-mode fiber, and Photon B is guided to the telescope and transmitted to Bob via an optical free-space link. The polarization of individual photons is analysed 

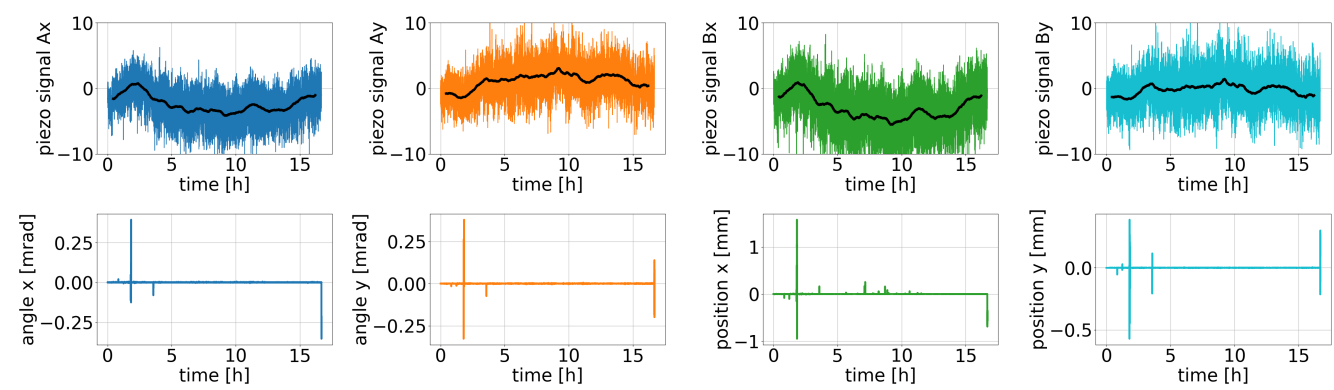

Figure 7. Overnight beam-stabilization. The plots in the top row show the closed-loop piezo signals and the plots in the bottom row show the corresponding residual errors of beam's angle and centroid. The solid black line is a 5-minute moving average, indicating the long-term changes in the system beam path.

and detection events are timestamped with respect to a local Rubidium clock. Timetags and measurement basis information has been sent via an RF-channel from Bob to Alice, whereby residual clock drift is compensated for by continuous tracking the two-photon correlation peak. Using QKD post-processing modules (Austrian Institute of Technology), key material was generated at a rates of tens of kbits/s and an average quantum bit error rate (QBER) of 3\% - depending on link conditions. The key is loaded into encryption- and decryption modules (Rohde\&Schwarz), which were used to establish a secure P2P-Network.

\section{SUMMARY}

A robust and reliable link infrastructure that allows for flexible exchange of quantum sources, detectors and transceiver telescopes is crucial for a fast transition from lab experiments into industry. The experimental platforms described in this paper are a possible way for a direct connection between the developing phase of quantum communication devices and existing communication networks. We have shown a concept, an opto-mechanical design and a demonstrator of multi-wavelengths transceiver breadboards with high performance mirror telescopes to handle various quantum source and receiver devices. Furthermore, we have shown the development of the PhotonBox as a low-cost lab container as well as the design for the QuBus. A high-performance mobile platform for ad-hoc test campaigns in quantum communications.

\section{ACKNOWLEDGMENTS}

This research is part of QuNET, an initiative of the German Federal Ministry of Education and Research (BMBF) and supported by funds from the research framework "Self-determined and secure in the digital world 2015-2020.

\section{REFERENCES}

[1] Steinlechner, F., de Vries, O., Fleischmann, N., Wille, E., Beckert, E., and Ursin, R., "Development of a space-proof polarization-entangled photon source," in [Conference on Lasers and Electro-Optics], Conference on Lasers and Electro-Optics, FTu1C.8, Optical Society of America (2016).

[2] Bennett, C. H. and Brassard, G., "Quantum cryptography: Public key distribution and coin tossing," Theoretical Computer Science 560, 7-11 (Dec 2014).

[3] Bennett, C. H., Brassard, G., and Mermin, N. D., "Quantum cryptography without bell's theorem," Phys. Rev. Lett. 68, 557-559 (Feb 1992). 